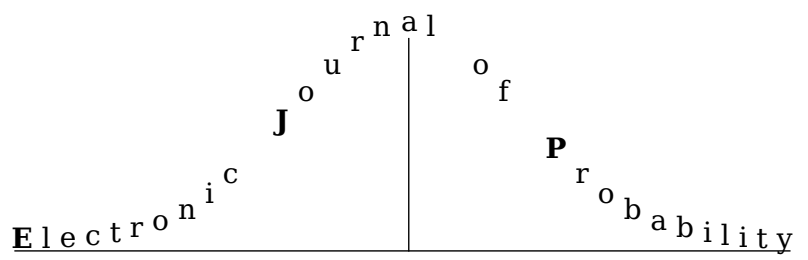

Electron. J. Probab. 25 (2020), article no. 115, 1-20.

ISSN: 1083-6489 https://doi.org/10.1214/20-EJP520

\title{
Fractional extreme distributions
}

\author{
Lotfi Boudabsa* Thomas Simon ${ }^{\dagger} \quad$ Pierre Vallois $^{\ddagger}$
}

\begin{abstract}
We consider three classes of linear differential equations on distribution functions, with a fractional order $\alpha \in[0,1]$. The integer case $\alpha=1$ corresponds to the three classical extreme families. In general, we show that there is a unique distribution function solving these equations, whose underlying random variable is expressed in terms of an exponential random variable and an integral transform of an independent $\alpha$-stable subordinator. From the analytical viewpoint, this distribution is in one-to-one correspondence with a Kilbas-Saigo function for the Weibull and Fréchet cases, and with a Le Roy function for the Gumbel case.
\end{abstract}

Keywords: Double Gamma function; extreme distribution; fractional differential equation; Kilbas-Saigo function; Le Roy function; stable subordinator.

MSC2020 subject classifications: 26A33; 33E12; 45E10; 60E05; 60G52.

Submitted to EJP on July 31, 2019, final version accepted on September 5, 2020.

\section{Introduction and statement of the main results}

The classical Fisher-Tippett-Gnedenko theorem states that the limit distributions arising from $a_{n}\left(\max \left(X_{1}, \ldots, X_{n}\right)-b_{n}\right)$ with $a_{n}>0, b_{n} \in \mathbb{R}$ and $\left(X_{1}, \ldots, X_{n}\right)$ an i.i.d. real sample, can be classified up to positive affine transformation into three families:

$$
\begin{cases}\mathbf{W}_{\rho}=\mathbf{L}^{1 / \rho} & \text { (Weibull distribution) } \\ \mathbf{F}_{\rho}=\mathbf{L}^{-1 / \rho} & \text { (Fréchet distribution) } \\ \mathbf{G}=\log \mathbf{L} & \text { (Gumbel distribution) }\end{cases}
$$

where $\mathbf{L}$ is the unit exponential random variable, $\rho$ is a positive parameter and, with an abuse of notation which we will make throughout the paper, we have identified a random variable with its law. From the distribution function viewpoint, the three

\footnotetext{
*Institut de Mathématiques, Ecole Polytechnique Fédérale de Lausanne, CH-1015 Lausanne, Switzerland. E-mail: lotfi.boudabsa@epfl.ch

${ }^{\dagger}$ Laboratoire Paul Painlevé, UMR 8524, Université de Lille, Cité Scientifique, F-59655 Villeneuve d’Ascq Cedex, France. E-mail: thomas.simon@univ-lille. $\mathrm{fr}$

${ }^{\ddagger}$ Institut de Mathématiques Elie Cartan, INRIA-BIGS, Université de Lorraine, BP 239, F-54506 Vandœuvrelès-Nancy Cedex, France. E-mail: pierre.vallois@univ-lorraine.fr
} 
above extreme laws can also be obtained as the unique solution to a certain ordinary differential equation. More precisely, if $F(x)$ stands for a distribution function on $\mathbb{R}$ and $\bar{F}(x)=1-F(x)$ denotes its associated survival function, the following equations

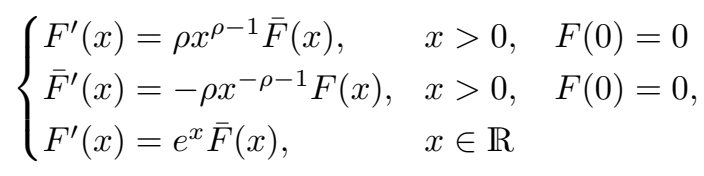

have each a unique solution which is respectively given by the Weibull distribution $\mathbf{W}_{\rho}$, the Fréchet distribution $\mathbf{F}_{\rho}$ and the Gumbel distribution $\mathbf{G}$.

Notice that since the derivative of $\bar{F}$ is $-F^{\prime}$, and vice versa, those three equations involve a logarithmic derivative and are solved via the exponential function. In this paper, we will consider some extensions of these equations in the context of fractional calculus. There are many types of fractional operators and throughout the paper we shall only consider Liouville fractional integrals and derivatives, which are the most common ones - see the Appendix A for all precise definitions and notations. In Liouville fractional calculus, the classical Mittag-Leffler function

$$
E_{\alpha}(z)=\sum_{n \geq 0} \frac{z^{n}}{\Gamma(1+n \alpha)}, \quad \alpha>0, z \in \mathbb{C},
$$

which can be viewed as a generalization of the exponential function, plays a fundamental role. We refer to Chapter 3 in [12] for a modern account on this function, and also to Chapter 2 therein for an interesting historical overview.

Let us first discuss an example. It is well-known from the general results of Barrett [3] - see also Lemma 3.24 and the inversion formula (E.1.10) in [12] - that for every $\alpha, \lambda>0$ the function $x \mapsto E_{\alpha}\left(-\lambda x^{\alpha}\right)$ solves on $\mathbb{R}^{+}$the following fractional differential equation

$$
\mathrm{D}_{0+}^{\alpha}(1-f)(x)=\lambda f(x),
$$

where $\mathrm{D}_{0+}^{\alpha}$ is the progressive Liouville fractional derivative on the half-axis. Besides, it follows from the works of Pillai [17] that for every $\alpha \in(0,1]$ the function $x \mapsto E_{\alpha}\left(-x^{\alpha}\right)$ is the survival function of a distribution on $\mathbb{R}^{+}$. More precisely, one has

$$
E_{\alpha}\left(-x^{\alpha}\right)=\mathbb{E}\left[e^{-x^{\alpha} \mathbf{Z}_{\alpha}^{-\alpha}}\right]=\mathbb{P}\left[\mathbf{Z}_{\alpha} \times \mathbf{L}^{\frac{1}{\alpha}}>x\right]
$$

where $\mathbf{Z}_{\alpha}$ has a standard positive $\alpha$-stable distribution with the normalization $\mathbb{E}\left[e^{-x \mathbf{Z}_{\alpha}}\right]=$ $e^{-x^{\alpha}}$ and, here and throughout, each terms of the product are assumed to be independent. This shows that the distribution function of the random variable $\mathbf{Z}_{\alpha} \times \mathbf{L}^{\frac{1}{\alpha}}$ solves the fractional differential equation

$$
\mathrm{D}_{0+}^{\alpha} F(x)=\bar{F}(x)
$$

on $(0, \infty)$, with the initial condition $F(0)=0$.

The above fact can be used to display another, dual example involving the regressive Liouville fractional derivative on the half-axis $\mathrm{D}_{-}^{\alpha}$ and the generalized Mittag-Leffler function

$$
E_{\alpha, \beta}(z)=\sum_{n \geq 0} \frac{z^{n}}{\Gamma(\beta+n \alpha)}, \quad \alpha, \beta>0, z \in \mathbb{C} .
$$

On the one hand, it follows indeed from the formulas (4.10.13) and (E.2.6) in [12] that for every $\alpha, \lambda>0$ the function $x \mapsto \Gamma(\alpha) E_{\alpha, \alpha}\left(-\lambda x^{-\alpha}\right)$ solves on $(0, \infty)$ the fractional differential equation

$$
\mathrm{D}_{-}^{\alpha}(1-f)(x)=\lambda x^{-2 \alpha} f(x) .
$$


On the other hand, the above Pillai result shows that for every $\alpha \in(0,1]$, the function

$$
\Gamma(\alpha) E_{\alpha, \alpha}\left(-x^{-\alpha}\right)=\Gamma(1+\alpha) E_{\alpha}^{\prime}\left(-x^{-\alpha}\right)=\mathbb{P}\left[\left(\mathbf{Z}_{\alpha}^{-1}\right)^{(\alpha)} \times \mathbf{L}^{-\frac{1}{\alpha}} \leq x\right]
$$

is a distribution function on $\mathbb{R}^{+}$, where the second equality follows from an elementary transformation of (1.2) involving the size-bias $\left(\mathbf{Z}_{\alpha}^{-1}\right)^{(\alpha)}$ of order $\alpha$ of the inverse positive $\alpha$-stable random variable. Recall that for $t \in \mathbb{R}$ and a positive random variable $X$ such that $\mathbb{E}\left[X^{t}\right]<\infty$ the size-bias of order $t$ of $X$ is the random variable $X^{(t)}$ whose law is defined by

$$
\mathbb{E}\left[f\left(X^{(t)}\right)\right]=\frac{\mathbb{E}\left[X^{t} f(X)\right]}{\mathbb{E}\left[X^{t}\right]}
$$

for all $f: \mathbb{R}^{+} \rightarrow \mathbb{R}$ bounded continuous, and that $\mathbb{E}\left[\mathbf{Z}_{\alpha}^{-\alpha}\right]=1 / \Gamma(1+\alpha)$. All of this shows that the distribution function of the random variable $\left(\mathbf{Z}_{\alpha}^{-1}\right)^{(\alpha)} \times \mathbf{L}^{-1 / \alpha}$ solves the fractional differential equation

$$
\mathrm{D}_{-}^{\alpha} \bar{F}(x)=x^{-2 \alpha} F(x)
$$

on $(0, \infty)$, with the initial condition $F(0)=0$.

In this paper, we wish to study more general fractional equations than (1.3) and (1.4), which are natural extensions of the above differential equations characterizing the classical extreme distributions. Our findings involve the $\alpha$-stable subordinator $\left\{\sigma_{t}^{(\alpha)} t \geq 0\right\}$, which is the real Lévy process starting from zero such that $\sigma_{1}^{(\alpha)} \stackrel{d}{=} \mathbf{Z}_{\alpha}$. For every $\alpha \in[0,1]$, its Laplace transform is given by

$$
\mathbb{E}\left[e^{-\lambda \sigma_{t}^{(\alpha)}}\right]=e^{-t \lambda^{\alpha}}, \quad \lambda, t \geq 0 .
$$

Observe that $\sigma^{(\alpha)}$ is a pure drift for $\alpha=1$ that is $\sigma_{t}^{(1)}=t$, and a pure killing at an exponential time $\mathbf{L}$ for $\alpha=0$ that is $\sigma_{t}^{(0)}=\infty$ on $\{t \geq \mathbf{L}\}$ and $\sigma_{t}^{(0)}=0$ on $\{t<\mathbf{L}\}$. Our first main result gives a fractional extension of the Weibull distribution.

Theorem 1.1. For every $\lambda, \rho>0$ and $\alpha \in[0,1]$, there exists a unique distribution function solving the fractional differential equation

$$
\mathrm{D}_{0+}^{\alpha} F(x)=\lambda x^{\rho-\alpha} \bar{F}(x)
$$

on $(0, \infty)$, with the initial condition $F(0)=0$. The corresponding random variable is

$$
\mathbf{W}_{\alpha, \lambda, \rho} \stackrel{d}{=} \mathbf{W}_{\rho} \times\left(\lambda \int_{0}^{\infty}\left(\left(1-\sigma_{t}^{(\alpha)}\right)_{+}\right)^{\rho-\alpha} d t\right)^{-\frac{1}{\rho}}
$$

In the above statement, we have used the standard notation $x_{+}=\max (x, 0)$ for $x \in \mathbb{R}$. Observe that the integral on the right-hand side is finite a.s. for every $\rho>0$ and $\alpha \in[0,1]$ : this is clear for $\alpha=1$ since $\rho>0$, and when $\alpha<1$ this follows from the fact that $\sigma^{(\alpha)}$ is a non-decreasing càdlàg process which crosses the level 1 a.s. by a jump - see e.g. Theorem III.4 in [5]. The above result shows that the fractional index $\alpha \in[0,1]$ of the derivative $\mathrm{D}_{0+}^{\alpha}$ gives rise to a non-trivial multiplicative perturbation of the Weibull random variable $\mathbf{W}_{\rho}$ expressed as the power of a certain Riemannian integral of the stable subordinator, whereas the parameter $\lambda$ is simply a scaling constant with $\mathbf{W}_{\alpha, \lambda, \rho} \stackrel{d}{=} \lambda^{-1 / \rho} \mathbf{W}_{\alpha, 1, \rho}$. One has also the identities

$$
\mathbf{W}_{1, \rho, \rho} \stackrel{d}{=} \mathbf{W}_{\rho} \quad \text { and } \quad \mathbf{W}_{0,1, \rho} \stackrel{d}{=} \frac{\mathbf{W}_{\rho}}{\mathbf{W}_{\rho}}
$$

where, here and throughout, each terms of the quotient are assumed to be independent. The random variable on the right has a Pareto distribution of type III - see [2] for a 
study of the latter distribution, and the mapping in law $\alpha \mapsto \mathbf{W}_{\alpha, \rho^{\alpha}, \rho}$ can be viewed as a parametrized arc connecting this Pareto III distribution and the Weibull distribution, the parameter being the index of the underlying stable subordinator.

Our second main result gives a fractional extension of the Fréchet distribution.

Theorem 1.2. For every $\lambda, \rho>0$ and $\alpha \in[0,1]$, there exists a unique distribution function solving the fractional differential equation

$$
\mathrm{D}_{-}^{\alpha} \bar{F}(x)=\lambda x^{-\rho-\alpha} F(x)
$$

on $(0, \infty)$, with the initial condition $F(0)=0$. The corresponding random variable is

$$
\mathbf{F}_{\alpha, \lambda, \rho} \stackrel{d}{=} \mathbf{F}_{\rho} \times\left(\lambda \int_{0}^{\infty}\left(1+\sigma_{t}^{(\alpha)}\right)^{-\rho-\alpha} d t\right)^{\frac{1}{\rho}} .
$$

In the above statement the integral on the right-hand side is finite a.s. by the law of the iterated logarithm at infinity for $\sigma^{(\alpha)}$ - see e.g. Theorem III.11 in [5]. As above, the index $\alpha$ of the derivative $\mathrm{D}_{-}^{\alpha}$ produces a multiplicative perturbation of the Fréchet random variable $\mathbf{F}_{\rho}$ via a Riemannian integral of $\sigma^{(\alpha)}$, whereas the parameter $\lambda$ is a scaling constant with $\mathbf{F}_{\alpha, \lambda, \rho} \stackrel{d}{=} \lambda^{1 / \rho} \mathbf{F}_{\alpha, 1, \rho}$. One has also the identities

$$
\mathbf{F}_{1, \rho, \rho} \stackrel{d}{=} \mathbf{F}_{\rho} \quad \text { and } \quad \mathbf{F}_{0,1, \rho} \stackrel{d}{=} \frac{\mathbf{F}_{\rho}}{\mathbf{F}_{\rho}} \stackrel{d}{=} \frac{\mathbf{W}_{\rho}}{\mathbf{W}_{\rho}} .
$$

It is interesting to observe from the two above theorems that the two mappings in law

$$
\alpha \mapsto \mathbf{L}^{ \pm \frac{1}{\rho}} \times\left(\rho^{\alpha} \int_{0}^{\infty}\left(1 \mp \sigma_{t}^{(\alpha)}\right)_{+}^{ \pm \rho-\alpha} d t\right)^{\mp \frac{1}{\rho}},
$$

with a sign switch at $\alpha=0$ corresponding to the trivial equation $F=x^{\rho} \bar{F}$, produce a parametrized arc connecting the classical extreme random variables $\mathbf{W}_{\rho}$ and $\mathbf{F}_{\rho}$. The traditional role of the $\alpha$-stable subordinator is to define a fractional Laplacian via the underlying subordinated semi-group whose marginals are the symmetric $\beta$-stable distributions with $\beta=2 \alpha$, the densities of the latter being up to some multiplicative constant the solutions to the fractional Cauchy problem

$$
\frac{\partial f}{\partial t}=\frac{\partial^{\beta} f}{\partial x^{\beta}}
$$

on $(0, \infty) \times \mathbb{R}^{+}$- see [11] and the references therein for more on this standard subject. The above results show that the $\alpha$-stable subordinator is also involved, by means of its Riemannian integrals, in the solution to some fractional differential equations naturally associated to the Weibull or the Fréchet distribution.

The classical Gumbel distribution is the limit in law of either $\rho\left(\mathbf{W}_{\rho}-1\right) \stackrel{d}{\longrightarrow} \mathbf{G}$ or $\rho\left(1-\mathbf{F}_{\rho}\right) \stackrel{d}{\longrightarrow} \mathbf{G}$ as $\rho \rightarrow \infty$. In order to define a fractional Gumbel distribution, it is natural from the above to introduce the random variable

$$
\mathbf{G}_{\alpha}=\log \left(\int_{0}^{\infty} e^{-\sigma_{t}^{(\alpha)}} d t\right)
$$

for every $\alpha \in[0,1]$, the a.s. convergence of the integral being a well-known consequence of

$$
\mathbb{E}\left[\int_{0}^{\infty} e^{-\sigma_{t}^{(\alpha)}} d t\right]=\int_{0}^{\infty} \mathbb{E}\left[e^{-\sigma_{t}^{(\alpha)}}\right] d t=\int_{0}^{\infty} e^{-t} d t=1 .
$$

We then have the following result involving the progressive Liouville fractional derivative on the line $\mathrm{D}_{+}^{\alpha}$, which can be guessed at the formal limit $\rho \rightarrow \infty$ after a logarithmic change of variable in Theorem 1.1 or Theorem 1.2, and whose derivation is actually rigorous. 
Theorem 1.3. For every $\lambda>0$ and $\alpha \in[0,1]$, there exists a unique distribution function solving the fractional differential equation

$$
\mathrm{D}_{+}^{\alpha} F(x)=\lambda^{\alpha} e^{\lambda x} \bar{F}(x)
$$

on $\mathbb{R}$. The corresponding random variable is

$$
\mathbf{G}_{\alpha, \lambda} \stackrel{d}{=} \lambda^{-1}\left(\mathbf{G}-\mathbf{G}_{\alpha}\right) .
$$

Unlike the fractional Weibull or Fréchet distributions, the perturbation on the standard Gumbel distribution induced by the parameter $\alpha$ of the progressive derivative $\mathrm{D}_{+}^{\alpha}$ is linear and not multiplicative. Again, the parameter $\lambda$ is a scaling constant with $\mathbf{G}_{\alpha, \lambda} \stackrel{d}{=} \lambda^{-1} \mathbf{G}_{\alpha, 1}$. One has also the identities

$$
\mathbf{G}_{1,1} \stackrel{d}{=} \mathbf{G} \quad \text { and } \quad \mathbf{G}_{0,1} \stackrel{d}{=} \mathbf{G}-\mathbf{G} .
$$

The random variable on the right has a standard logistic distribution - see [18] for an account on the latter distribution, and the mapping in law $\alpha \mapsto \mathbf{G}_{\alpha, 1}$ can be viewed as a parametrized arc connecting the logistic distribution and the Gumbel distribution.

The proof of the three above theorems is divided in two parts. In Section 2 we prove the uniqueness of the solutions to a more general class of fractional equations. These results have an independent interest, because the uniqueness problem is not always addressed in the literature on fractional calculus. It is classical in analysis to show that the solution of a differential equation must be the fixed point of an integral equation, and we use the same method, in the framework of fractional calculus. In Section 3 we show the existence of a probability law solving the above equations, and we establish the explicit multiplicative, respectively additive, factorizations. This is done via a one-to-one correspondence with a Kilbas-Saigo function, respectively a Le Roy function. These two unusual special functions lead to a family of positive random variables characterized by their entire moments and previously studied in [16], in a more general context. The results of [16] imply then the required factorizations with an exponential random variable and an independent stable subordinator.

\section{Some uniqueness results on fractional hazard rates}

In this section we prove the uniqueness of distribution functions solving fractional equations of the type (1.5), (1.6) or (1.7), where the power function is replaced by a more general hazard rate. We repeat that all definitions and notations on the fractional operators that we will consider here can be found in Appendix A.

\subsection{The Weibull case}

We consider the equation

$$
\mathrm{D}_{0+}^{\alpha} F=h \bar{F}, \quad F(0)=0,
$$

where $F$ is a distribution function and $h:(0, \infty) \rightarrow \mathbb{R}^{+}$is measurable and locally bounded. In the case $\alpha=1$, there exists a solution to (2.1) if and only if

$$
\int_{0}^{\infty} h(t) d t=\infty
$$

with a unique solution given by

$$
\bar{F}(x)=\exp \left\{-\int_{0}^{x} h(t) d t\right\} .
$$


Recall that the function $h$ is called either the reliability function or the hazard rate of the underlying positive random variable. In the case $\alpha=0$, there exists a solution to (2.1) if and only if $h$ is non-decreasing, $h(0)=0$ and $h(x) \rightarrow \infty$ as $x \rightarrow \infty$, with a unique solution given by

$$
\bar{F}(x)=\frac{1}{1+h(x)} .
$$

In order to state our result in the fractional case $\alpha \in(0,1)$, we introduce the following linear operator

$$
A_{0+}^{\alpha, h}: f \mapsto \mathrm{I}_{0+}^{\alpha}(h f)
$$

which is well-defined on measurable functions from $(0, \infty)$ to $\mathbb{R}^{+}$.

Theorem 2.1. Assume that there exists $\rho>0$ such that $h(x)=O\left(x^{\rho-\alpha}\right)$ as $x \rightarrow 0$. Then, if it exists, the distribution function satisfying (2.1) is uniquely defined by the convergent series

$$
\bar{F}(x)=\sum_{n \geq 0}(-1)^{n}\left(A_{0+}^{\alpha, h}\right)^{n} \mathbf{1}(x), \quad x>0 .
$$

Proof. We begin by transforming (2.1) into an integral equation. Since $F$ is a distribution function on $\mathbb{R}^{+}$, there exists a probability measure $\mu$ on $\mathbb{R}^{+}$such that

$$
F(x)=\int_{0}^{x} \mu(d t)=\mathrm{I}_{0+}^{\alpha}\left(F_{\alpha, \mu}\right)(x), \quad x>0
$$

where we have set

$$
F_{\alpha, \mu}(x)=\frac{1}{\Gamma(1-\alpha)} \int_{0}^{x}(x-t)^{-\alpha} \mu(d t), \quad x>0,
$$

and the second equality in (2.3) is a direct consequence of Fubini's theorem and of the evaluation of the Beta integral of the first kind. Moreover, it is easy to see that the function $F_{\alpha, \mu}$, which might take infinite values, is nevertheless locally integrable at zero since $\mu$ is a probability. Hence, applying $\mathrm{I}_{0+}^{\alpha}$ on both sides of (2.1), we can use the inversion formula (A.1) and get

$$
F=\mathrm{I}_{0+}^{\alpha}(h \bar{F})=A_{0+}^{\alpha, h}(\bar{F})
$$

on $(0, \infty)$. This leads to the fixed point equation $\bar{F}=\mathbf{1}-A_{0+}^{\alpha, h}(\bar{F})$ and, by the linearity of $A_{0+}^{\alpha, h}$, to

$$
\bar{F}(x)=\sum_{k=0}^{n-1}(-1)^{k}\left(A_{0+}^{\alpha, h}\right)^{k} \mathbf{1}(x)+(-1)^{n}\left(A_{0+}^{\alpha, h}\right)^{n}(\bar{F})(x), \quad x>0,
$$

for every $n \geq 1$. Fixing now $x>0$, the assumption made on $h$ implies that there exists a constant $c>0$ such that $h(t) \leq c t^{\rho-\alpha}$ for every $t \in[0, x]$. Since moreover $\bar{F}(t) \leq 1$ for every $t \in[0, x]$, an immediate induction based on the Beta integral of the first kind implies

$$
0 \leq\left(A_{0+}^{\alpha, h}\right)^{n}(\bar{F})(x) \leq\left(\prod_{k=1}^{n} \frac{\Gamma(1-\alpha+k \rho)}{\Gamma(1+k \rho)}\right) c^{n} x^{\rho n} \rightarrow 0 \text { as } n \rightarrow \infty,
$$

where the convergence towards zero follows e.g. from (1.1.5) in [1]. This completes the proof.

Remark 2.2. (a) It can be proved that the series defined in (2.2) converges uniformly on every compact set and that it tends to 1 as $x \rightarrow 0$. On the other hand, because of the alternate signs it is difficult to guess whether it is non-increasing and converges to 
zero as $x \rightarrow \infty$. In the particular case when $h$ is a power function, Theorem 1.1 gives a positive answer with the help of a Kilbas-Saigo function and the $\alpha$-stable subordinator. It would be interesting to know if there are other hazard rate functions $h$ such that the series in (2.2) is indeed a survival function on $(0, \infty)$.

(b) In a different direction, sharing a certain analogy with the previous item, the authors have introduced in $[19,20]$ generalized fractional distributions which are not conventional and classical distributions with fractional hazard rates. In [21], the stochastic approximation of fractional probability distribution have been studied.

(c) The above proof shows the more general fact that under the same assumption on $h$, for every $\alpha \in(0,1)$ there exists a unique bounded solution to

$$
\mathrm{D}_{0+}^{\alpha}(f(0)-f)=h f
$$

on $(0, \infty)$, which is given by

$$
f=f(0) \times \sum_{n \geq 0}(-1)^{n}\left(A_{0+}^{\alpha, h}\right)^{n} \mathbf{1} .
$$

This can be viewed as an extension of (1.1) which handles the case when $h$ is a positive constant.

\subsection{The Fréchet case}

We consider the equation

$$
\mathrm{D}_{-}^{\alpha} \bar{F}=h F, \quad F(0)=0,
$$

given on distribution functions, where $h:(0, \infty) \rightarrow \mathbb{R}^{+}$is measurable and locally bounded. In the case $\alpha=1$, there exists a solution to (2.4) if and only if $h$ is locally integrable on $(0, \infty]$ and such that

$$
\int_{0}^{\infty} h(t) d t=\infty
$$

with a unique solution given by

$$
F(x)=\exp \left\{-\int_{x}^{\infty} h(t) d t\right\} .
$$

In the case $\alpha=0$, there exists a solution to (2.4) if and only if $h$ is non-increasing, $h(0+)=\infty$ and $h(x) \rightarrow 0$ as $x \rightarrow \infty$, with a unique solution given by

$$
F(x)=\frac{1}{1+h(x)}
$$

In order to state our result in the fractional case $\alpha \in(0,1)$, we introduce the following linear operator

$$
A_{-}^{\alpha, h}: f \mapsto \mathrm{I}_{-}^{\alpha}(h f),
$$

which is well-defined on measurable functions from $(0, \infty)$ to $\mathbb{R}^{+}$.

Theorem 2.3. Assume that there exists $\rho>0$ such that $h(x)=O\left(x^{-\rho-\alpha}\right)$ as $x \rightarrow \infty$. Then, if it exists, the distribution function satisfying (2.4) is uniquely defined by the convergent series

$$
F(x)=\sum_{n \geq 0}(-1)^{n}\left(A_{-}^{\alpha, h}\right)^{n} \mathbf{1}(x), \quad x>0 .
$$


Proof. The proof is analogous to that of (2.3), except that we deal with survival functions. Since $\bar{F}$ is a survival function on $\mathbb{R}^{+}$, there exists a probability measure $\mu$ on $\mathbb{R}^{+}$such that

$$
\bar{F}(x)=\int_{x}^{\infty} \mu(d t)=\mathrm{I}_{-}^{\alpha}\left(\bar{F}_{\alpha, \mu}\right)(x), \quad x>0,
$$

where we have set

$$
\bar{F}_{\alpha, \mu}(x)=\frac{1}{\Gamma(1-\alpha)} \int_{x}^{\infty}(t-x)^{-\alpha} \mu(d t), \quad x>0,
$$

and used Fubini's theorem together with the evaluation of the Beta integral of the second kind. The function $\bar{F}_{\alpha, \mu}$ is locally integrable at infinity since for every $y>0$ one has

$$
\int_{y}^{\infty} \bar{F}_{\alpha, \mu}(x) d x=\frac{1}{\Gamma(2-\alpha)} \int_{y}^{\infty}(t-y)^{1-\alpha} \mu(d t)=\frac{1}{\Gamma(2-\alpha)} \int_{y}^{\infty}(s-y)^{-\alpha} \bar{F}(s) d s<\infty,
$$

the finiteness of the third integral following from the fact that $\mathrm{D}_{-}^{\alpha} \bar{F}$ must be finite on $(0, \infty)$. Hence, we can apply the inversion formula (A.2) and get $\bar{F}=\mathrm{I}_{-}^{\alpha}(h F)=A_{-}^{\alpha, h}(F)$ on $(0, \infty)$, which leads to $F=\mathbf{1}-A_{-}^{\alpha, h}(F)$ and then to

$$
F(x)=\sum_{k=0}^{n-1}(-1)^{k}\left(A_{-}^{\alpha, h}\right)^{k} \mathbf{1}(x)+(-1)^{n}\left(A_{-}^{\alpha, h}\right)^{n}(F)(x), \quad x>0,
$$

for every $n \geq 1$. Fixing now $x>0$, the assumption made on $h$ implies that there exists a constant $c>0$ such that $h(t) \leq c t^{-\rho-\alpha}$ for every $t>x$. Since moreover $F(t) \leq 1$ for every $t>x$, an induction based on the Beta integral of the second kind implies

$$
0 \leq\left(A_{-}^{\alpha, h}\right)^{n}(F)(x) \leq\left(\prod_{k=1}^{n} \frac{\Gamma(k \rho)}{\Gamma(\alpha+k \rho)}\right) c^{n} x^{-\rho n} \rightarrow 0 \quad \text { as } n \rightarrow \infty,
$$

which completes the proof.

Remark 2.4. (a) It can be proved that the series defined in (2.5) converges uniformly on every compact set of $(0, \infty)$ and that it tends to 1 as $x \rightarrow \infty$. As above, it is not easy to guess from the alternate signs whether the series is non-decreasing and converges to zero as $x \rightarrow 0$. The case when $h$ is a power function gives a positive answer in Theorem 1.2, with the help of another Kilbas-Saigo function. It would be interesting to know if there are other functions $h$ such that the series in (2.5) is indeed a distribution function on $(0, \infty)$.

(b) The above proof shows that under the same assumption on $h$, for every $\alpha \in(0,1)$ there exists a unique bounded function having a limit $\ell$ at infinity and solving

$$
\mathrm{D}_{-}^{\alpha}(\ell-f)=h f
$$

on $(0, \infty)$, which is given by

$$
f=\ell \times \sum_{n \geq 0}(-1)^{n}\left(A_{-}^{\alpha, h}\right)^{n} \mathbf{1} .
$$

Observe that this solution is zero if $\ell=0$. 


\subsection{The Gumbel case}

We consider the equation

$$
\mathrm{D}_{+}^{\alpha} F=h \bar{F}, \quad F(x)>0 \text { on } \mathbb{R}
$$

given on distribution functions, where $h: \mathbb{R} \mapsto \mathbb{R}^{+}$is measurable and locally bounded. In the case $\alpha=1$, there exists a solution to (2.6) if and only if $h$ is locally integrable at $-\infty$ and such that

$$
\int_{\mathbb{R}} h(t) d t=\infty
$$

with a unique solution given by

$$
\bar{F}(x)=\exp \left\{-\int_{-\infty}^{x} h(t) d t\right\} .
$$

In the case $\alpha=0$, there exists a solution to (2.1) if and only if $h$ is non-decreasing, $h(x) \rightarrow 0$ as $x \rightarrow-\infty$ and $h(x) \rightarrow \infty$ as $x \rightarrow \infty$, with a unique solution given by

$$
\bar{F}(x)=\frac{1}{1+h(x)} .
$$

In order to state our result in the fractional case $\alpha \in(0,1)$, we introduce the following linear operator

$$
A_{+}^{\alpha, h}: f \mapsto \mathrm{I}_{+}^{\alpha}(h f),
$$

which is well-defined on measurable functions from $\mathbb{R}$ to $\mathbb{R}^{+}$. The following result is a simple variation on Theorem 2.3.

Theorem 2.5. Assume that there exists $\lambda>0$ such that $h(-x)=O\left(e^{-\lambda x}\right)$ as $x \rightarrow \infty$. Then, if it exists, the distribution function satisfying (2.6) is uniquely defined by the convergent series

$$
\bar{F}(x)=\sum_{n \geq 0}(-1)^{n}\left(A_{+}^{\alpha, h}\right)^{n} \mathbf{1}(x), \quad x \in \mathbb{R} .
$$

Proof. Setting $G(x)=\bar{F}(-x)$, we see that $G$ is a distribution function on $\mathbb{R}$ with $\bar{G}(x)=$ $F(-x)$. Changing the variable transforms (2.6) into

$$
\mathrm{D}_{-}^{\alpha} \bar{G}=g G, \quad G(x)>0 \text { on } \mathbb{R},
$$

with $g(x)=h(-x)$. An argument similar to the proof of Theorem 2.3, where the evaluation of the Beta integral of the second kind is replaced by that of the Gamma integral, gives then the unique solution

$$
G(x)=\sum_{n \geq 0}(-1)^{n}\left(A_{-}^{\alpha, g}\right)^{n} \mathbf{1}(x), \quad x \in \mathbb{R} .
$$

Changing the variable backwards, we obtain the required (2.7).

\section{Proof of the main theorems}

In this section we show the existence of the real random variables associated to the fractional differential equations (1.5), (1.6) and (1.7), and we express them in terms of the unit exponential random variable and an integral transform of an independent $\alpha$-stable subordinator. An important ingredient in the proof is the following infinite independent product

$$
\mathbf{T}(a, b, c)=\prod_{n \geq 0}\left(\frac{a+n b+c}{a+n b}\right) \mathbf{B}_{a+n b, c}
$$


where, here and throughout, $\mathbf{B}_{a, b}$ denotes a standard Beta random variable. We refer to Section 2.1 in [16] for more details on this product, including the fact that it is a.s. convergent for every $a, b, c>0$. We also mention from Proposition 2 in [16] that its Mellin transform is

$$
\mathbb{E}\left[\mathbf{T}(a, b, c)^{s}\right]=\left(\frac{\Gamma\left(a b^{-1}\right)}{\Gamma\left((a+c) b^{-1}\right)}\right)^{s} \times \frac{[a+c ; b]_{s}}{[a ; b]_{s}}
$$

for every $s>-a$, where $[z ; \delta]_{s}$ stands for the generalized Pochhammer symbol which is defined in (B.3) below. In general, we shall refer to the Appendix B for all the properties of Barnes' double Gamma function and its associated Pochhammer symbol that we will need.

The other important tools are the aforementioned Kilbas-Saigo and Le Roy functions. The Kilbas-Saigo functions are three-parameter generalizations of the classical MittagLeffler functions $E_{\alpha}$ and $E_{\alpha, \beta}$, defined by the convergent series representation

$$
E_{\alpha, m, l}(z)=\sum_{n \geq 0}\left(\prod_{k=1}^{n} \frac{\Gamma(1+\alpha((k-1) m+l))}{\Gamma(1+\alpha((k-1) m+l+1))}\right) z^{n}, \quad z \in \mathbb{C},
$$

for $\alpha, m>0$ and $l>-1 / \alpha$, with the convention made here and throughout that an empty product always equals 1 . Note that $E_{\alpha}=E_{\alpha, 1,0}$ and that $\Gamma(\beta) E_{\alpha, \beta}=E_{\alpha, 1, \frac{\beta-1}{\alpha}}$. We refer to Chapter 5.2 in [12] for an extensive account, including an extension to complex values of the parameter $l$. The Le Roy functions are simple generalizations of the exponential function, defined by

$$
\mathcal{L}_{\alpha}(z)=\sum_{n \geq 0} \frac{z^{n}}{(n !)^{\alpha}}, \quad z \in \mathbb{C},
$$

for $\alpha>0$. Introduced in [15] in the context of analytic continuation, Le Roy functions are much less studied than Mittag-Leffler's - see however the recent paper [10] and the references therein.

\subsection{Proof of Theorem 1.1}

We first consider the case $\alpha \in(0,1)$. The uniqueness is a direct consequence of Theorem 2.1 with $h(x)=\lambda x^{\rho-\alpha}$. Moreover, we know by (2.2) that a distribution solving (1.5), if it exists, has survival function

$$
\bar{F}(x)=\sum_{n \geq 0}(-1)^{n}\left(A_{0+}^{\alpha, h}\right)^{n} \mathbf{1}(x), \quad x>0 .
$$

Since

$$
\left(\mathrm{I}_{0+}^{\alpha} t^{\beta}\right)(x)=\frac{\Gamma(\beta+1)}{\Gamma(\alpha+\beta+1)} x^{\alpha+\beta+1}
$$

for every $\beta>-1$, an induction implies

$$
\bar{F}(x)=\sum_{n \geq 0}\left(\prod_{k=1}^{n} \frac{\Gamma(k \rho+1-\alpha)}{\Gamma(k \rho+1)}\right)\left(-\lambda x^{\rho}\right)^{n}=E_{\alpha, \frac{\rho}{\alpha}, \frac{\rho}{\alpha}-1}\left(-\lambda x^{\rho}\right)
$$

for every $x \geq 0$. Observe that alternatively, the fact that $E_{\alpha, \frac{\rho}{\alpha}, \frac{\rho}{\alpha}-1}\left(-\lambda x^{\rho}\right)$ is a solution to (1.5) follows from Theorem 5.27 and the inversion formula (E.1.10) in [12].

It thus remains to prove that $x \mapsto E_{\alpha, \frac{\rho}{\alpha}, \frac{\rho}{\alpha}-1}\left(-\lambda x^{\rho}\right)$ is a survival function on $\mathbb{R}^{+}$and to identify the underlying positive random variable. For every $z \in \mathbb{C}$, one has

$$
E_{\alpha, \frac{\rho}{\alpha}, \frac{\rho}{\alpha}-1}(z)=\sum_{n \geq 0} a_{n}(\alpha, \rho) \frac{z^{n}}{n !}
$$


with

$$
a_{n}(\alpha, \rho)=\rho^{-n} \prod_{k=1}^{n} \frac{\Gamma(k \rho+1-\alpha)}{\Gamma(k \rho)} .
$$

Let us now consider the positive random variable

$$
\frac{\Gamma(\rho+1-\alpha)}{\Gamma(\rho+1)} \mathbf{T}\left(1, \rho^{-1},(1-\alpha) \rho^{-1}\right) .
$$

By the aforementioned Proposition 2 in [16] and the concatenation formula (B.1), the positive entire moments of the latter random variable are given by

$$
\delta^{n}\left(\frac{[1+(1-\alpha) \delta ; \delta]_{n}}{[1 ; \delta]_{n}}\right)=a_{n}(\alpha, \rho)
$$

where $\delta=\rho^{-1}$. The Stirling formula (B.2) implies

$$
a_{n}(\alpha, \rho)^{-\frac{1}{2 n}} \sim \kappa n^{\frac{\alpha-1}{2}} \quad \text { as } n \rightarrow \infty
$$

for some positive constant $\kappa$, so that Carleman's criterion

$$
\sum_{n \geq 1} a_{n}(\alpha, \rho)^{-\frac{1}{2 n}}=\infty
$$

is fulfilled, and the law of the latter random variable is determined by its positive entire moments. Finally, it follows from Theorem (b) (i) in [16] with $q=\rho-\alpha$ that

$$
\frac{\Gamma(\rho+1-\alpha)}{\Gamma(\rho+1)} \mathbf{T}\left(1, \rho^{-1},(1-\alpha) \rho^{-1}\right) \stackrel{d}{=} \mathcal{A}(\alpha, 0, \rho-\alpha) \stackrel{d}{=} \int_{0}^{\infty}\left(1-\sigma_{t}^{(\alpha)}\right)_{+}^{\rho-\alpha} d t .
$$

All in all, we have shown that

$$
E_{\alpha, \frac{\rho}{\alpha}, \frac{\rho}{\alpha}-1}(z)=\mathbb{E}\left[\exp \left\{z \int_{0}^{\infty}\left(1-\sigma_{t}^{(\alpha)}\right)_{+}^{\rho-\alpha} d t\right\}\right], \quad z \in \mathbb{C} .
$$

This implies

$$
\begin{aligned}
E_{\alpha, \frac{\rho}{\alpha}, \frac{\rho}{\alpha}-1}\left(-\lambda x^{\rho}\right) & =\mathbb{E}\left[\exp \left\{-\lambda x^{\rho} \int_{0}^{\infty}\left(1-\sigma_{t}^{(\alpha)}\right)_{+}^{\rho-\alpha} d t\right\}\right] \\
& =\mathbb{P}\left[\mathbf{L}>x^{\rho}\left(\lambda \int_{0}^{\infty}\left(1-\sigma_{t}^{(\alpha)}\right)_{+}^{\rho-\alpha} d t\right)\right]=\mathbb{P}\left[\mathbf{W}_{\alpha, \lambda, \rho}>x\right]
\end{aligned}
$$

for every $x \geq 0$, which completes the proof for $\alpha \in(0,1)$. The case $\alpha=1$ is that of the classical Weibull distribution and was already discussed in the introduction. Finally, the case $\alpha=0$ amounts to solving $F=\lambda x^{\rho}(1-F)$, which yields $\bar{F}(x)=1 /\left(1+\lambda x^{\rho}\right)$ and

$$
\mathbf{W}_{0, \lambda, \rho} \stackrel{d}{=}\left(\frac{\mathbf{L}}{\lambda \mathbf{L}}\right)^{\frac{1}{\rho}} \stackrel{d}{=} \mathbf{W}_{\rho} \times\left(\lambda \int_{0}^{\infty}\left(1-\sigma_{t}^{(0)}\right)_{+}^{\rho} d t\right)^{-\frac{1}{\rho}} .
$$

Remark 3.1. (a) The main result of [13] implies the identification

$$
\left(\frac{\Gamma(\rho+1-\alpha)}{\Gamma(\rho)} \mathbf{T}\left(1, \rho^{-1},(1-\alpha) \rho^{-1}\right)\right)^{-\frac{1}{\rho}} \stackrel{d}{=} \mathcal{G}(\rho+1-\alpha, 1-\alpha)
$$

where $\mathcal{G}(m, a)$ is the generalized stable random variable with parameters $m>a>0$, whose density is up to normalization the unique positive solution to

$$
\mathrm{I}_{0+}^{a} f=x^{m} f
$$


on $(0, \infty)$. This yields the further identity in law

$$
\mathbf{W}_{\alpha, \lambda, \rho} \stackrel{d}{=}\left(\rho \lambda^{-1}\right)^{\frac{1}{\rho}} \mathbf{W}_{\rho} \times \mathcal{G}(\rho+1-\alpha, 1-\alpha) .
$$

In particular - see the introduction in [13] for the third identity, one has

$$
\mathbf{W}_{\alpha, 1, \alpha} \stackrel{d}{=} \alpha^{1 / \alpha} \mathbf{W}_{\alpha, \alpha, \alpha} \stackrel{d}{=}\left(\alpha^{1 / \alpha} \mathcal{G}(1,1-\alpha)\right) \times \mathbf{W}_{\alpha} \stackrel{d}{=} \mathbf{Z}_{\alpha} \times \mathbf{L}^{1 / \alpha},
$$

in accordance with the Pillai distribution mentioned in the introduction since

$$
\mathbb{P}\left[\mathbf{W}_{\alpha, 1, \alpha}>x\right]=E_{\alpha, 1,0}\left(-x^{\alpha}\right)=E_{\alpha}\left(-x^{\alpha}\right), \quad x \geq 0 .
$$

Notice that the integro-differential equation (3.2) shares some formal similarities with (1.5). Nevertheless it is essentially different because it deals with densities whereas (1.5) deals with distribution functions.

(b) There exist unique solutions to fractional differential equations of the type (1.5) without the restriction to distribution functions. The main result of [4] states that for every $\alpha \in(0,1]$, there is a unique solution to

$$
(\alpha+1) \mathrm{D}_{0+}^{\alpha} f=-x f
$$

satisfying the boundary condition

$$
f(x) \sim \frac{x^{\alpha-1}}{\Gamma(\alpha) \Gamma(1 /(\alpha+1))}
$$

at zero, which is the density function of the running maximum of a spectrally positive $(\alpha+1)$-stable Lévy process starting from zero.

(c) With the above notation, one has

$$
a_{n}(\alpha, \rho)=\frac{n !}{\Phi_{\alpha, \rho}(1) \cdots \Phi_{\alpha, \rho}(n)}
$$

with

$$
\Phi_{\alpha, \rho}(x)=\frac{\Gamma(1+\rho x)}{\Gamma(1-\alpha+\rho x)}=\frac{1}{\Gamma(1-\alpha)}+\frac{\alpha}{\rho \Gamma(1-\alpha)} \int_{0}^{\infty}\left(1-e^{-x u}\right) \frac{e^{-u \rho^{-1}}}{\left(1-e^{-u \rho^{-1}}\right)^{\alpha+1}} d u
$$

a Bernstein function. By Proposition 3.3 in [8], we deduce

$$
\frac{\Gamma(\rho+1-\alpha)}{\Gamma(\rho+1)} \mathbf{T}\left(1, \rho^{-1},(1-\alpha) \rho^{-1}\right) \stackrel{d}{=} \int_{0}^{\infty} e^{-\xi_{t}^{(\alpha, \rho)}} d t
$$

where $\left\{\xi_{t}^{(\alpha, \rho)}, t \geq 0\right\}$ is the subordinator having Laplace exponent

$$
\mathbb{E}\left[e^{-\lambda \xi_{t}^{(\alpha, \rho)}}\right]=e^{-t \Phi_{\alpha, \rho}(\lambda)}
$$

This leads to

$$
\mathbf{W}_{\alpha, \lambda, \rho} \stackrel{d}{=} \mathbf{W}_{\rho} \times\left(\lambda \int_{0}^{\infty} e^{-\xi_{t}^{(\alpha, \rho)}} d t\right)^{-\frac{1}{\rho}}
$$

Let us notice that the identification (3.3) can also be deduced from Corollary 5 in [16] is the case $q=\rho-\alpha>-\alpha$ and $\hat{\rho}=1$, with the notation therein. Observe finally that this is consistent with the limiting case $\alpha=1$ with $\xi_{t}^{(1, \rho)}=\rho t$ and $\alpha=0$ with $\xi_{t}^{(0, \rho)}=\sigma_{t}^{(0)}$.

(d) The above proof shows that the function

$$
x \mapsto E_{\alpha, m, m-1}(-x)
$$


is completely monotone (CM) for every $\alpha \in(0,1]$ and $m>0$. This can be viewed as a generalization of the classic result by Pollard that $E_{\alpha}(-x)=E_{\alpha, 1,0}(-x)$ is CM for every $\alpha \in(0,1]$ - see e.g. Proposition 3.23 in [12]. This also solves a conjecture stated in [9] see Section 4 and equations (10) and (11) therein. Notice that the formula (3.1) implies the Bernstein representation

$$
E_{\alpha, m, m-1}(-x)=\mathbb{E}\left[\exp -x\left\{\int_{0}^{\infty}\left(1-\sigma_{t}^{(\alpha)}\right)_{+}^{\alpha(m-1)} d t\right\}\right], \quad x \geq 0
$$

For $m=1$, setting $T_{\alpha}=\inf \left\{t>0, \sigma_{t}^{(\alpha)}>1\right\} \stackrel{d}{=} \mathbf{Z}_{\alpha}^{-\alpha}$ we obtain

$$
E_{\alpha}(-x)=E_{\alpha, 1,0}(-x)=\mathbb{E}\left[e^{-x T_{\alpha}}\right]=\mathbb{E}\left[e^{-x \mathbf{Z}_{\alpha}^{-\alpha}}\right],
$$

a well-known fact following from our discussion prior to the statement of Theorem 1.1. In a forthcoming paper, we will generalize this fact and show some further analytical properties of the Kilbas-Saigo function $E_{\alpha, m, m-1}$.

We end this section with a convergent series representation, in the non-explicit case $\alpha \in(0,1)$, for the density $f_{\alpha, \lambda, \rho}^{\mathbf{W}}$ of $\mathbf{W}_{\alpha, \lambda, \rho}$. This is an immediate consequence of a term-by-term differentiation of the survival function

$$
\mathbb{P}\left[\mathbf{W}_{\alpha, \lambda, \rho}>x\right]=E_{\alpha, \frac{\rho}{\alpha}, \frac{\rho}{\alpha}-1}\left(-\lambda x^{\rho}\right)
$$

which was obtained during the proof of Theorem 1.1.

Corollary 3.2. For every $\alpha \in(0,1)$, the density of $\mathbf{W}_{\alpha, \lambda, \rho}$ has the following convergent series representation on $(0, \infty)$

$$
f_{\alpha, \lambda, \rho}^{\mathbf{W}}(x)=\lambda x^{\rho-1} \sum_{n \geq 0}\left(\prod_{j=0}^{n} \frac{\Gamma(j \rho+\rho+1-\alpha)}{\Gamma(j \rho+\rho)}\right) \frac{\left(-\lambda x^{\rho}\right)^{n}}{\rho^{n} n !} .
$$

\subsection{Proof of Theorem 1.2}

The proof is analogous to that of Theorem 1.1, except that we will deal with distribution functions instead of survival functions. We begin with the case $\alpha \in(0,1)$ and the uniqueness follows from Theorem 2.3. Besides, we know by (2.5) that a distribution solving (1.6), if it exists, must have distribution function

$$
F(x)=\sum_{n \geq 0}(-1)^{n}\left(A_{-}^{\alpha, h}\right)^{n} \mathbf{1}(x), \quad x>0,
$$

with $h(x)=\lambda x^{-\rho-\alpha}$. Since

$$
\left(\mathrm{I}_{-}^{\alpha} t^{-\beta}\right)(x)=\frac{\Gamma(\beta-\alpha)}{\Gamma(\beta)} x^{\alpha-\beta}
$$

for every $\beta>\alpha$, an induction implies

$$
F(x)=\sum_{n \geq 0}\left(\prod_{k=1}^{n} \frac{\Gamma(k \rho)}{\Gamma(k \rho+\alpha)}\right)\left(-\lambda x^{-\rho}\right)^{n}=E_{\alpha, \frac{\rho}{\alpha}, \frac{\rho-1}{\alpha}}\left(-\lambda x^{-\rho}\right)
$$

for every $x>0$. Alternatively, the fact that $E_{\alpha, \frac{\rho}{\alpha}, \frac{\rho-1}{\alpha}}\left(-\lambda x^{-\rho}\right)$ is a solution to (1.6) follows from Theorem 5.30 and the inversion formula (E.1.10) in [12]. It remains to prove that $x \mapsto E_{\alpha, \frac{\rho}{\alpha}, \frac{\rho-1}{\alpha}}\left(-\lambda x^{-\rho}\right)$ is a distribution function on $(0, \infty)$ and to identify the underlying positive random variable. For every $z \in \mathbb{C}$, one has

$$
E_{\alpha, \frac{\rho}{\alpha}, \frac{\rho-1}{\alpha}}(z)=\sum_{n \geq 0} b_{n}(\alpha, \rho) \frac{z^{n}}{n !}
$$


with

$$
b_{n}(\alpha, \rho)=\rho^{-n} \prod_{k=1}^{n} \frac{\Gamma(k \rho+1)}{\Gamma(k \rho+\alpha)} .
$$

Reasoning exactly as above implies that $\left\{b_{n}(\alpha, \rho), n \geq 0\right\}$ is the determinate integer moment sequence of the positive random variable

$$
\frac{\Gamma(\rho)}{\Gamma(\rho+\alpha)} \mathbf{T}\left(1+\alpha \rho^{-1}, \rho^{-1},(1-\alpha) \rho^{-1}\right) \stackrel{d}{=} \int_{0}^{\infty}\left(1+\sigma_{t}^{(\alpha)}\right)^{-\rho-\alpha} d t,
$$

where the identity in law follows from Corollary 3 in [16]. We have hence shown that

$$
E_{\alpha, \frac{\rho}{\alpha}, \frac{\rho-1}{\alpha}}(z)=\mathbb{E}\left[\exp \left\{z \int_{0}^{\infty}\left(1+\sigma_{t}^{(\alpha)}\right)^{-\rho-\alpha} d t\right\}\right], \quad z \in \mathbb{C} .
$$

This implies

$$
\begin{aligned}
E_{\alpha, \frac{\rho}{\alpha}, \frac{\rho-1}{\alpha}}\left(-\lambda x^{-\rho}\right) & =\mathbb{E}\left[\exp \left\{-\lambda x^{-\rho} \int_{0}^{\infty}\left(1+\sigma_{t}^{(\alpha)}\right)^{-\rho-\alpha} d t\right\}\right] \\
& =\mathbb{P}\left[\mathbf{L} \geq x^{-\rho}\left(\lambda \int_{0}^{\infty}\left(1+\sigma_{t}^{(\alpha)}\right)^{-\rho-\alpha} d t\right)\right]=\mathbb{P}\left[\mathbf{F}_{\alpha, \lambda, \rho} \leq x\right]
\end{aligned}
$$

for every $x>0$, which completes the proof for $\alpha \in(0,1)$. As for Theorem 1.2 the remaining cases $\alpha=0$ and $\alpha=1$ are elementary and we leave the details to the reader.

Remark 3.3. (a) Contrary to $\mathbf{W}_{\alpha, \lambda, \rho}$, the factor

$$
\int_{0}^{\infty}\left(1+\sigma_{t}^{(\alpha)}\right)^{-\rho-\alpha} d t
$$

appearing in the decomposition of $\mathbf{F}_{\alpha, \lambda, \rho}$ cannot be expressed as a generalized stable law. On the other hand, this factor can also be viewed as the perpetuity of some subordinator: rewriting

$$
b_{n}(\alpha, \rho)=\frac{n !}{\Psi_{\alpha, \rho}(1) \cdots \Psi_{\alpha, \rho}(n)}
$$

with the Bernstein function

$$
\Psi_{\alpha, \rho}(x)=\frac{\Gamma(\alpha+\rho x)}{\Gamma(\rho x)}=\frac{\alpha}{\rho \Gamma(1-\alpha)} \int_{0}^{\infty}\left(1-e^{-x u}\right) \frac{e^{-u \rho^{-1}}}{\left(1-e^{-u \rho^{-1}}\right)^{\alpha+1}} d u
$$

we obtain as above

$$
\frac{\Gamma(\rho)}{\Gamma(\rho+\alpha)} \mathbf{T}\left(1+\alpha \rho^{-1}, \rho^{-1},(1-\alpha) \rho^{-1}\right) \stackrel{d}{=} \int_{0}^{\infty} e^{-\zeta_{t}^{(\alpha, \rho)}} d t
$$

where $\left\{\zeta_{t}^{(\alpha, \rho)}, t \geq 0\right\}$ is the subordinator having Laplace exponent

$$
\mathbb{E}\left[e^{-\lambda \zeta_{t}^{(\alpha, \rho)}}\right]=e^{-t \Psi_{\alpha, \rho}(\lambda)} .
$$

This leads to

$$
\mathbf{F}_{\alpha, \lambda, \rho} \stackrel{d}{=} \mathbf{F}_{\rho} \times\left(\lambda \int_{0}^{\infty} e^{-\zeta_{t}^{(\alpha, \rho)}} d t\right)^{\frac{1}{\rho}} .
$$

Let us again notice that the identification (3.5) can be deduced from Corollary 5 in [16] is the case $q=-\rho-\alpha<-\alpha$ and $\hat{\rho}=0-$ see also Remark 10 therein. Observe also that 
the limiting cases $\alpha=0$ and $\alpha=1$ are consistent, with respectively $\zeta_{t}^{(0, \rho)}=\sigma_{t}^{(0)}$ and $\zeta_{t}^{(1, \rho)}=\rho t$.

(b) Since $\Psi_{1-\alpha, \rho}(x) \Phi_{\alpha, \rho}(x)=\rho x$, we have

$$
n !=\rho^{n} \times b_{n}(1-\alpha, \rho) \times a_{n}(\alpha, \rho)
$$

for all $\alpha \in[0,1], \rho>0$ and $n \geq 1$. By moment determinacy, this implies the following factorization of the unit exponential law

$$
\begin{aligned}
\mathbf{L} & \stackrel{d}{=}\left(\int_{0}^{\infty} \rho\left(1+\sigma_{t}^{(1-\alpha)}\right)^{-\rho-1+\alpha} d t\right) \times\left(\int_{0}^{\infty}\left(\left(1-\sigma_{t}^{(\alpha)}\right)_{+}\right)^{\rho-\alpha} d t\right) \\
& \stackrel{d}{=}\left(\int_{0}^{\infty}\left(1+\rho^{-1} \sigma_{t}^{(1-\alpha)}\right)^{-\rho-1+\alpha} d t\right) \times\left(\int_{0}^{\infty}\left(\left(1-\rho^{-1} \sigma_{t}^{(\alpha)}\right)_{+}\right)^{\rho-\alpha} d t\right)
\end{aligned}
$$

which is valid for all $\alpha \in[0,1]$ and $\rho>0$. For $\rho=\alpha$, this factorization reads

$$
\mathbf{L} \stackrel{d}{=}\left(\int_{0}^{\infty} \frac{\alpha d t}{1+\sigma_{t}^{(1-\alpha)}}\right) \times \mathbf{Z}_{\alpha}^{-\alpha} \stackrel{d}{=} \mathbf{L}^{\alpha} \times \mathbf{Z}_{\alpha}^{-\alpha}
$$

where the first identity follows from Remark 3.1 (d) and the second one from (3.4) in [16]. The simple identity $\mathbf{L} \stackrel{d}{=} \mathbf{L}^{\alpha} \times \mathbf{Z}_{\alpha}^{-\alpha}$ is well-known as Shanbhag-Sreehari's identity. It has been thoroughly discussed in Section 3 of [6] from the point of view of perpetuities of subordinators, and their associated remainders. Observe also that changing the variable and letting $\rho \rightarrow \infty$ in (3.6) leads to

$$
\mathbf{L} \stackrel{d}{=}\left(\int_{0}^{\infty} e^{-\sigma_{t}^{(1-\alpha)}} d t\right) \times\left(\int_{0}^{\infty} e^{-\sigma_{t}^{(\alpha)}} d t\right)
$$

another classic identity obtained in [8] - see Example E therein. Last, it is interesting to mention the following identity, which follows at once from (3.6), Theorem 1.1 and Theorem 1.2:

$$
\mathbf{W}_{\alpha, 1, \rho} \times \mathbf{F}_{1-\alpha, \rho, \rho}^{-1} \stackrel{d}{=}\left(\frac{\mathbf{L} \times \mathbf{L}}{\mathbf{L}}\right)^{\frac{1}{\rho}} .
$$

(c) The above proof shows that the function

$$
x \mapsto E_{\alpha, m, m-\frac{1}{\alpha}}(-x)
$$

is $\mathrm{CM}$ for every $\alpha \in(0,1]$ and $m>0$. This is a generalization of the fact that $E_{\alpha, 1,1-\frac{1}{\alpha}}(-x)=\Gamma(\alpha) E_{\alpha, \alpha}(-x)$ is CM for every $\alpha \in(0,1]$, which is itself a direct consequence of the aforementioned Pollard theorem because $\alpha E_{\alpha}^{\prime}(-x)=E_{\alpha, \alpha}(-x)$. The formula (3.4) implies the Bernstein representation

$$
E_{\alpha, m, m-\frac{1}{\alpha}}(-x)=\mathbb{E}\left[\exp -x\left\{\int_{0}^{\infty}\left(1+\sigma_{t}^{(\alpha)}\right)^{-\alpha(m+1)} d t\right\}\right], \quad x \geq 0 .
$$

For $m=1$, with the notation of Remark 3.1 (d) we obtain

$$
E_{\alpha, 1,1-\frac{1}{\alpha}}(-x)=\Gamma(\alpha) E_{\alpha, \alpha}(-x)=\Gamma(1+\alpha) E_{\alpha}^{\prime}(-x)=\Gamma(1+\alpha) \mathbb{E}\left[T_{\alpha} e^{-x T_{\alpha}}\right]=\mathbb{E}\left[e^{-x T_{\alpha}^{(1)}}\right]
$$

where $T_{\alpha}^{(1)}$ is the size-bias of order 1 of $T_{\alpha}$. This implies the curious identity

$$
T_{\alpha}^{(1)} \stackrel{d}{=} \int_{0}^{\infty}\left(1+\sigma_{t}^{(\alpha)}\right)^{-2 \alpha} d t
$$


In a different direction, is is worth recalling that for every $\beta>\alpha$ and $\alpha \in(0,1]$ the function

$$
E_{\alpha, 1, \frac{\beta-1}{\alpha}}(-x)=\Gamma(\beta) E_{\alpha, \beta}(-x)=\mathbb{E}\left[e^{-x \mathbf{B}_{\alpha, \beta-\alpha}^{\alpha} \times T_{\alpha}^{(1)}}\right]
$$

is also $\mathrm{CM}$, where the Bernstein representation involving the $\alpha$-power of a standard Beta distribution follows directly from Lemma 4.26 in [12] - see also the references therein. In a companion paper, we will come back to this example together with further analytical properties of the Kilbas-Saigo functions $E_{\alpha, m, m-\frac{1}{\alpha}}$.

We end this section with a convergent series representation in the non-explicit case $\alpha \in(0,1)$ for the density $f_{\alpha, \lambda, \rho}^{\mathbf{F}}$ of $\mathbf{F}_{\alpha, \lambda, \rho}$. This is a consequence of a term-by-term differentiation of the distribution function

$$
\mathbb{P}\left[\mathbf{F}_{\alpha, \lambda, \rho} \leq x\right]=E_{\alpha, \frac{\rho}{\alpha}, \frac{\rho-1}{\alpha}}\left(-\lambda x^{-\rho}\right)
$$

which was obtained during the proof of Theorem 1.2.

Corollary 3.4. For every $\alpha \in(0,1)$, the density of $\mathbf{F}_{\alpha, \lambda, \rho}$ has the following convergent series representation on $(0, \infty)$

$$
f_{\alpha, \lambda, \rho}^{\mathbf{F}}(x)=\lambda x^{-\rho-1} \sum_{n \geq 0}\left(\prod_{j=0}^{n} \frac{\Gamma(j \rho+\rho+1)}{\Gamma(j \rho+\rho+\alpha)}\right) \frac{\left(-\lambda x^{-\rho}\right)^{n}}{\rho^{n} n !} .
$$

\subsection{Proof of Theorem 1.3}

The argument is shorter than for Theorems 1.1 and 1.2. We first consider the case $\alpha \in(0,1)$. The uniqueness is a direct consequence of Theorem 2.5. We next compute, by Fubini's theorem, the survival function

$$
\mathbb{P}\left[\mathbf{G}_{\alpha, \lambda}>x\right]=\mathbb{P}\left[\mathbf{L}>e^{\lambda x+\mathbf{G}_{\alpha}}\right]=\mathbb{E}\left[e^{-e^{\lambda x+\mathbf{G}_{\alpha}}}\right]=\sum_{n \geq 0} \frac{(-1)^{n} e^{\lambda n x}}{n !} \mathbb{E}\left[e^{n \mathbf{G}_{\alpha}}\right]
$$

for every $x \in \mathbb{R}$. On the other hand, since

$$
e^{\mathbf{G}_{\alpha}} \stackrel{d}{=} \int_{0}^{\infty} e^{-\sigma_{t}^{(\alpha)}} d t
$$

we know from Proposition 3.3 in [8] that $\mathbb{E}\left[e^{n \mathbf{G}_{\alpha}}\right]=(n !)^{1-\alpha}$ for all $n \geq 0$. This implies

$$
\bar{F}(x)=\mathbb{P}\left[\mathbf{G}_{\alpha, \lambda}>x\right]=\sum_{n \geq 0} \frac{(-1)^{n} e^{\lambda n x}}{(n !)^{\alpha}}=\mathcal{L}_{\alpha}\left(-e^{\lambda x}\right) .
$$

A direct integration based on the Gamma integral and Fubini's theorem shows finally that

$$
\mathrm{D}_{+}^{\alpha} F(x)=\lambda^{\alpha} e^{\lambda x} \bar{F}(x)
$$

for every $x \in \mathbb{R}$ as required. The case $\alpha=1$ was already discussed in the introduction with a unique solution $F(x)=\mathbb{P}[\mathbf{G} \leq \lambda x]=\mathbb{P}\left[\mathbf{G}_{1, \lambda} \leq x\right]$, whereas the unique solution in the case $\alpha=0$ is obviously $F(x)=1 /\left(1+e^{-\lambda x}\right)$, which is the distribution function of $\lambda^{-1}(\mathbf{G}-\mathbf{G})=\mathbf{G}_{0, \lambda}$.

Remark 3.5. (a) The above proof also shows that the unique distribution function solving the fractional differential equation

$$
\mathrm{D}_{-}^{\alpha} \bar{F}(x)=\lambda^{\alpha} e^{-\lambda x} F(x), \quad F(x)>0 \text { on } \mathbb{R},
$$

is $F(x)=\mathcal{L}_{\alpha}\left(-e^{-\lambda x}\right)=\mathbb{P}\left[-\mathbf{G}_{\alpha, \lambda} \leq x\right]$. 
(b) It is easy to deduce from the representations of the fractional extreme distributions in terms of integrals of the stable subordinator the following convergences in law

$$
\rho \lambda^{-1}\left(\mathbf{W}_{\alpha, \rho^{\alpha}, \rho}-1\right) \stackrel{d}{\longrightarrow} \mathbf{G}_{\alpha, \lambda} \quad \text { and } \quad \rho \lambda^{-1}\left(1-\mathbf{F}_{\alpha, \rho^{\alpha}, \rho}\right) \stackrel{d}{\longrightarrow} \mathbf{G}_{\alpha, \lambda}
$$

as $\rho \rightarrow \infty$, for every $\alpha \in[0,1]$ and $\lambda>0$. Observe that the case $\alpha=\lambda=1$ amounts to the aforementioned convergences in law $\rho\left(\mathbf{W}_{\rho}-1\right) \stackrel{d}{\longrightarrow} \mathbf{G}$ and $\rho\left(1-\mathbf{F}_{\rho}\right) \stackrel{d}{\longrightarrow} \mathbf{G}$.

As above, we finish this paragraph with a convergent series representation in the non-explicit case $\alpha \in(0,1)$ for the density $f_{\alpha, \lambda}^{\mathbf{G}}$ of $\mathbf{G}_{\alpha, \lambda}$, which is a consequence of a term-by-term differentiation of the survival function $\mathbb{P}\left[\mathbf{G}_{\alpha, \lambda}>x\right]=\mathcal{L}_{\alpha}\left(-e^{\lambda x}\right)$.

Corollary 3.6. For every $\alpha \in(0,1]$, the density of $\mathbf{G}_{\alpha, \lambda}$ has the following convergent series representation on $\mathbb{R}$

$$
f_{\alpha, \lambda}^{\mathbf{G}}(x)=\sum_{n \geq 1} \frac{(-1)^{n-1} \lambda n e^{\lambda n x}}{(n !)^{\alpha}} .
$$

\section{A Fractional integrals and derivatives}

In this paragraph, we fix the notation on the fractional operators which are used throughout the paper. This is an excerpt from the beginning of Chapter 2 in [14]. We will consider only three kinds of such operators which are the most familiar ones, and our fractional parameter $\alpha$ will always be supposed in $[0,1]$. There are certainly many other fractional operators with a larger family of fractional parameters, and we refer to the whole Chapter 2 in [14] for an account.

\section{A.1 Progressive Liouville operators on the half-axis}

For every $\alpha \in(0,1)$, the operator $f \mapsto \mathrm{I}_{0+}^{\alpha}(f)$ with

$$
\mathrm{I}_{0+}^{\alpha}(f)(x)=\frac{1}{\Gamma(\alpha)} \int_{0}^{x}(x-u)^{\alpha-1} f(u) d u, \quad x>0,
$$

is well-defined, taking possibly infinite values, on measurable functions $f:(0, \infty) \rightarrow \mathbb{R}^{+}$. It is easy to see that if $f$ is integrable at zero, then so is $\mathrm{I}_{0+}^{\alpha}(f)$ - see Lemma 2.1 in [14] for a more general result. The corresponding fractional derivative $f \mapsto \mathrm{D}_{0+}^{\alpha} f$ is such that

$$
\mathrm{D}_{0+}^{\alpha}(f)(x)=\frac{\mathrm{d}}{\mathrm{d} x}\left(\mathrm{I}_{0+}^{1-\alpha}(f)\right)(x)
$$

and is well-defined almost everywhere as soon as $f=\mathrm{I}_{0+}^{\alpha}(g)$ for some $g$ integrable at zero. Moreover, for such functions there is an inversion formula

$$
\mathrm{I}_{0+}^{\alpha}\left(\mathrm{D}_{0+}^{\alpha}(f)\right)=\mathrm{I}_{0+}^{\alpha}(g)=f,
$$

which is valid almost everywhere - see Lemma 2.5 in [14]. These operators are extended to the boundary cases $\alpha=0$ with $\mathrm{I}_{0+}^{0}=\mathrm{D}_{0+}^{0}=\mathrm{Id}$ and $\alpha=1$ with $\mathrm{I}_{0+}^{1}$ and $\mathrm{D}_{0+}^{1}$ being respectively the usual running integral and derivative - see (2.1.7) in [14].

\section{A.2 Regressive Liouville operators on the half-axis}

For every $\alpha \in(0,1)$, the operator $f \mapsto \mathrm{I}_{-}^{\alpha}(f)$ with

$$
\mathrm{I}_{-}^{\alpha}(f)(x)=\frac{1}{\Gamma(\alpha)} \int_{x}^{\infty}(u-x)^{\alpha-1} f(u) d u, \quad x>0
$$


is well-defined on measurable functions $f:(0, \infty) \rightarrow \mathbb{R}^{+}$. It is easy to see that if $f$ is integrable at infinity, then so is $\mathrm{I}_{-}^{\alpha}(f)$ - see again Lemma 2.1 in [14]. The corresponding fractional derivative $f \mapsto \mathrm{D}_{-}^{\alpha} f$ is such that

$$
\mathrm{D}_{-}^{\alpha}(f)(x)=-\frac{\mathrm{d}}{\mathrm{d} x}\left(\mathrm{I}_{-}^{1-\alpha}(f)\right)(x)
$$

and is well-defined as soon as $f=\mathrm{I}_{-}^{\alpha}(g)$ for some $g$ integrable at infinity. Moreover, for such functions there is an inversion formula

$$
\mathrm{I}_{-}^{\alpha}\left(\mathrm{D}_{-}^{\alpha}(f)\right)=\mathrm{I}_{-}^{\alpha}(g)=f
$$

which is valid almost everywhere. These operators are extended to the boundary cases $\alpha=0$ with $\mathrm{I}_{-}^{0}=\mathrm{D}_{-}^{0}=\mathrm{Id}$ and $\alpha=1$ with $\mathrm{I}_{-}^{1}$ and $\mathrm{D}_{-}^{1}$ being respectively the usual running integral and the opposite of the usual derivative - see again (2.1.7) in [14].

\section{A.3 Progressive Liouville operators on the real axis}

For every $\alpha \in(0,1)$, the operator $f \mapsto \mathrm{I}_{+}^{\alpha}(f)$ with

$$
\mathrm{I}_{+}^{\alpha}(f)(x)=\frac{1}{\Gamma(\alpha)} \int_{-\infty}^{x}(x-u)^{\alpha-1} f(u) d u, \quad x \in \mathbb{R},
$$

is well-defined on measurable functions $f: \mathbb{R} \rightarrow \mathbb{R}^{+}$. Observe that $\mathrm{I}_{+}^{\alpha}(f)(-x)=\mathrm{I}_{-}^{\alpha}(g)(x)$ with $g(x)=f(-x)$ so that we can transfer to $\mathrm{I}_{+}^{\alpha}$ the properties on $\mathrm{I}_{-}^{\alpha}$. In particular, if $f$ is integrable at $-\infty$, then so is $\mathrm{I}_{+}^{\alpha}(f)$. The corresponding fractional derivative $f \mapsto \mathrm{D}_{+}^{\alpha} f$ is such that

$$
\mathrm{D}_{+}^{\alpha}(f)(x)=-\frac{\mathrm{d}}{\mathrm{d} x}\left(\mathrm{I}_{+}^{1-\alpha}(f)\right)(x)
$$

and is well-defined as soon as $f=\mathrm{I}_{+}^{\alpha}(g)$ for some $g$ integrable at $-\infty$. Moreover, for such functions there is an inversion formula $\mathrm{I}_{+}^{\alpha}\left(\mathrm{D}_{+}^{\alpha}(f)\right)=\mathrm{I}_{+}^{\alpha}(g)=f$, which is valid almost everywhere. These operators are extended to the boundary cases $\alpha=0$ with $\mathrm{I}_{+}^{0}=\mathrm{D}_{+}^{0}=\mathrm{Id}$ and $\alpha=1$ with $\mathrm{I}_{+}^{1}$ and $\mathrm{D}_{+}^{1}$ being respectively the usual running integral and derivative.

\section{B Barnes' double Gamma function}

In this paragraph, extracted from [7] to which we refer for further results, we collect a few facts about Barnes' double Gamma function $G(z ; \delta)$. For every $\delta>0$, this function is defined as the unique solution to the functional equation

$$
G(z+1 ; \delta)=\Gamma\left(z \delta^{-1}\right) G(z ; \delta)
$$

with normalization $G(1 ; \delta)=1$. This function is holomorphic on $\mathbb{C}$ and admits the following exponential representation

$$
G(z ; \delta)=\exp \int_{0}^{\infty}\left(\frac{1-e^{-z x}}{\left(1-e^{-x}\right)\left(1-e^{-\delta x}\right)}-\frac{z e^{-\delta x}}{1-e^{-\delta x}}+(z-1)\left(\frac{z}{2 \delta}-1\right) e^{-\delta x}-1\right) \frac{d x}{x}
$$

which is valid for $\Re(z)>0$ - see (5.1) in [7]. The following Stirling type asymptotic behaviour

$$
\log G(z ; \delta)-\frac{1}{2 \delta}\left(z^{2} \log z-\left(\frac{3}{2}+\log \delta\right) z^{2}-(1+\delta) z \log z\right)-A z-B \log z \rightarrow C
$$


is valid for $|z| \rightarrow \infty$ with $|\arg (z)|<\pi$, for some real constants $A, B$ and $C$ which are given in (4.5) of [7]. In this paper we make an extensive use of the following Pochhammer type symbol

$$
[a ; \delta]_{s}=\frac{G(a+s ; \delta)}{G(a ; \delta)}
$$

which is well-defined for every $a, \delta>0$ and $s>-a$.

\section{References}

[1] George E. Andrews, Richard Askey, and Ranjan Roy, Special functions, Encyclopedia of Mathematics and its Applications, vol. 71, Cambridge University Press, Cambridge, 1999. MR-1688958

[2] Barry C. Arnold, Christopher A. Robertson, and Hsiaw-Chan Yeh, Some properties of a Pareto-type distribution, Sankhyā Ser. A 48 (1986), no. 3, 404-408. MR-905450

[3] J. H. Barrett, Differential equations of non-integer order, Canad. J. Math. 6 (1954), 529-541. MR-64936

[4] Violetta Bernyk, Robert C. Dalang, and Goran Peskir, The law of the supremum of a stable Lévy process with no negative jumps, Ann. Probab. 36 (2008), no. 5, 1777-1789. MR-2440923

[5] Jean Bertoin, Lévy processes, Cambridge Tracts in Mathematics, vol. 121, Cambridge University Press, Cambridge, 1996. MR-1406564

[6] Jean Bertoin and Marc Yor, On subordinators, self-similar Markov processes and some factorizations of the exponential variable, Electron. Comm. Probab. 6 (2001), 95-106. MR1871698

[7] J. Billingham and A. C. King, Uniform asymptotic expansions for the Barnes double gamma function, Proc. Roy. Soc. London Ser. A 453 (1997), no. 1964, 1817-1829. MR-1478136

[8] Philippe Carmona, Frédérique Petit, and Marc Yor, On the distribution and asymptotic results for exponential functionals of Lévy processes, Exponential functionals and principal values related to Brownian motion, Bibl. Rev. Mat. Iberoamericana, Rev. Mat. Iberoamericana, Madrid, 1997, pp. 73-130. MR-1648657

[9] Edmundo Capelas de Oliveira, Francesco Mainardi, and Jayme Vaz, Jr., Fractional models of anomalous relaxation based on the Kilbas and Saigo function, Meccanica 49 (2014), no. 9, 2049-2060. MR-3240277

[10] Roberto Garrappa, Sergei Rogosin, and Francesco Mainardi, On a generalized threeparameter Wright function of Le Roy type, Fract. Calc. Appl. Anal. 20 (2017), no. 5, 11961215. MR-3721895

[11] R. Gorenflo and F. Mainardi, Fractional calculus and stable probability distributions, vol. 50, 1998, Fourth Meeting on Current Ideas in Mechanics and Related Fields (Kraków, 1997), pp. 377-388. MR-1648257

[12] Rudolf Gorenflo, Anatoly A. Kilbas, Francesco Mainardi, and Sergei V. Rogosin, Mittag-Leffler functions, related topics and applications, Springer Monographs in Mathematics, Springer, Heidelberg, 2014. MR-3244285

[13] Wissem Jedidi, Thomas Simon, and Min Wang, Density solutions to a class of integrodifferential equations, J. Math. Anal. Appl. 458 (2018), no. 1, 134-152. MR-3711897

[14] Anatoly A. Kilbas, Hari M. Srivastava, and Juan J. Trujillo, Theory and applications of fractional differential equations, North-Holland Mathematics Studies, vol. 204, Elsevier Science B.V., Amsterdam, 2006. MR-2218073

[15] É. Le Roy, Valeurs asymptotiques de certaines séries procédant suivant les puissances entières et positives d'une variable réelle., Bull. Sci. Math., II. Sér. 24 (1899), 245-268 (French).

[16] Julien Letemplier and Thomas Simon, On the law of homogeneous stable functionals, ESAIM Probab. Stat. 23 (2019), 82-111. MR-3922819

[17] R. N. Pillai, On Mittag-Leffler functions and related distributions, Ann. Inst. Statist. Math. 42 (1990), no. 1, 157-161. MR-1054728 
[18] F. W. Steutel, Infinite divisibility in theory and practice, Scand. J. Statist. 6 (1979), no. 2, 57-64. MR-538596

[19] Charles S. Tapiero and Pierre Vallois, Fractional randomness, Phys. A 462 (2016), 1161-1177. MR-3531632

[20] Charles S. Tapiero and Pierre Vallois, Implied fractional hazard rates and default risk distributions, Probab. Uncertain. Quant. Risk 2 (2017), Paper No. 2, 14. MR-3625731

[21] Charles S. Tapiero and Pierre Vallois, Fractional randomness and the Brownian bridge, Phys. A 503 (2018), 835-843. MR-3886808 